\title{
REALIZAÇÃO DE TESTES RÁPIDOS E VACINAÇÃO CONTRA O HPV EM ADOLESCENTES: DESAFIOS DA ATENÇÃO BÁSICA EM SAÚDE
}

\author{
Daiani Aparecida Stangherlin Frasson ${ }^{1}$; Juliana Silveira Colomé ${ }^{2}$
}

\section{RESUMO}

Devido a adolescência ser uma fase de transição com inúmeras questões psicológicas, sociais e fisiológicas, como o começo da vida sexual deve-se promover e vigiar a saúde desses indivíduos para prevenir e tratar possíveis Infecções Sexualmente Transmissíveis (IST's). O estudo caracteriza-se como exploratóriodescritivo, documental, estruturado em uma abordagem quantitativa. Realizada na região central do Rio Grande do Sul, entre maio de 2020 a maio de 2021. Resultando em 4.110 (100\%) testagem rápida entre a faixa etária dos 10 aos 14 anos e 15 aos 19 anos e $4.226(100,00 \%)$ administração de imunizantes contra o Papilomavírus Humano (HPV). Portanto, percebe uma grande demanda de usuários entre a faixa etária dos 15 aos 19 anos na procura da realização de testes rápidos nos serviços de saúde. Na questão de vacinação contra o HPV, ainda é pouco disseminada a sua importância, devendo ter mais campanhas durante 0 ano.

Palavras-chave: Enfermagem; Atenção Primária à Saúde; Sistema Único de Saúde; Promoção da Saúde.

Eixo Temático: Atenção Integral e Promoção à Saúde (AIPS).

\section{INTRODUÇÃO}

$\mathrm{Na}$ adolescência, consistindo como uma transição de uma criança para um adulto, ocorrem significativas mudanças psicológicas, sociais e fisiológicas como, por exemplo, a puberdade (POTTER, et al., 2018). Essa transformação, muitas vezes, ocorre de modo paralelo a muitas dificuldades, visto que alguns adolescentes se compararem constantemente e não têm com quem possam conversar sobre essas mudanças de vida. Nessa fase, observam-se, ainda, as primeiras experiências amorosas, o sentimento de independência e busca pela identidade

\footnotetext{
${ }_{1}^{1}$ Autora e Apresentadora - Universidade Franciscana - UFN, e-mail: daiani.frasson@ufn.edu.br

${ }^{2}$ Autora e Orientadora - Universidade Franciscana - UFN, e-mail: juliana@ufn.edu.br
} 
pessoal e pela aceitação à um grupo de amizades (SADOCK, B. J; SADOCK, V. A., 2007).

Maior parte dos adolescentes inicia a vida sexual destituído de informações, ocasionando muitos adolescentes a não fazerem uso de preservativos durante as reações sexuais. Sendo assim, se faz crucial estratégias de orientações sobre infecções sexualmente transmissíveis (IST's) e como prevenir e a importância da vacina do HPV. Assim como oferecer testes rápidos e aconselhamento a esses indivíduos (BRASIL, 2017).

Nesse sentido, uma estratégia para assistir esses indivíduos de forma mais próxima de suas vidas tem-se a Atenção Primária em Saúde (APS), que por meio dos profissionais e serviços de saúde tem potencial de apoiar, identificar riscos, vulnerabilidades e trabalhar através da promoção, prevenção, diagnóstico, tratamento, reabilitação, recuperação e redução de danos (BRASIL, 2012).

Desse modo, a instituição do Programa Saúde na Escola (PSE), em 2017, surge para auxiliar de forma a trabalhar em conjunto com os profissionais da educação para assistir os adolescentes no ambiente que eles já se sentem seguros e assim criando vínculo para o cuidado continuado (BRASIL, 2015).

No ano de 2010, o Estado do Rio Grande do Sul instituiu a Política Estadual de Atenção Integral a Saúde de Adolescentes (PEAISA). Ressaltando três eixos principais para trabalhar com esse público, sendo que um deles é a saúde sexual e reprodutiva como segundo eixo, compreendendo o adolescente autor da própria saúde englobando os direitos, planejamento familiar, acesso aos testes rápidos e estímulo para serem autores da própria saúde (RIO GRANDE DO SUL, 2010).

Dessa forma, analisar as ações e desafios na realização dos testes rápidos e aplicação da vacina contra HPV se faz necessária.

\section{METODOLOGIA}

O estudo caracteriza-se como exploratório-descritivo, documental, estruturado em uma abordagem quantitativa. A pesquisa foi realizada na Secretaria Municipal de Saúde, no Núcleo de Atenção Básica em Saúde, mais especificamente no setor de gestão da Política de Atenção à Saúde do Adolescente do município de Santa Maria. 
Os dados foram coletados por meio de um software (sistema MV) que integra todos os serviços do Sistema Único de Saúde no município. Cada variável foi coletada a cada mês em dois grupos de indivíduos, na faixa etária de 10 a 14 anos e 15 a 19 anos.

Os dados coletados são referentes à saúde do adolescente na APS, desenvolvidas no período de maio de 2020 a maio de 2021: realização de testes rápidos e imunização contra o Papilomavírus Humano (HPV).

Destaca-se que essa análise foi mencionada pela gestão municipal da política como uma ação pertinente e necessária, que ainda não havia sido realizada em função da alta demanda direcionada ao Núcleo de Atenção Básica no ano de 2020. O desenvolvimento do estudo considerou os aspectos éticos, conforme Resolução №. 466 de 12 de dezembro de 2012 do Conselho Nacional de Pesquisa, possuindo registro de aprovação número 3.093.323 (BRASIL, 2012).

\section{RESULTADOS E DISCUSSÕES}

Os testes rápidos incluídos na análise foram: HIV, HIV em gestantes, Sífilis e Sífilis em gestante, hepatite $B$ e hepatite $C$, entre dois grupos de 10 a 14 anos e 15 a 19 anos. No Gráfico 1 apresentam-se os dados conforme região geográficoadministrativa do município: 
Gráfico 1. Apresentação dos dados de testes rápidos realizados na APS em adolescentes conforme região geográfico-administrativa do município de Santa Maria - RS, 2020 - 2021.

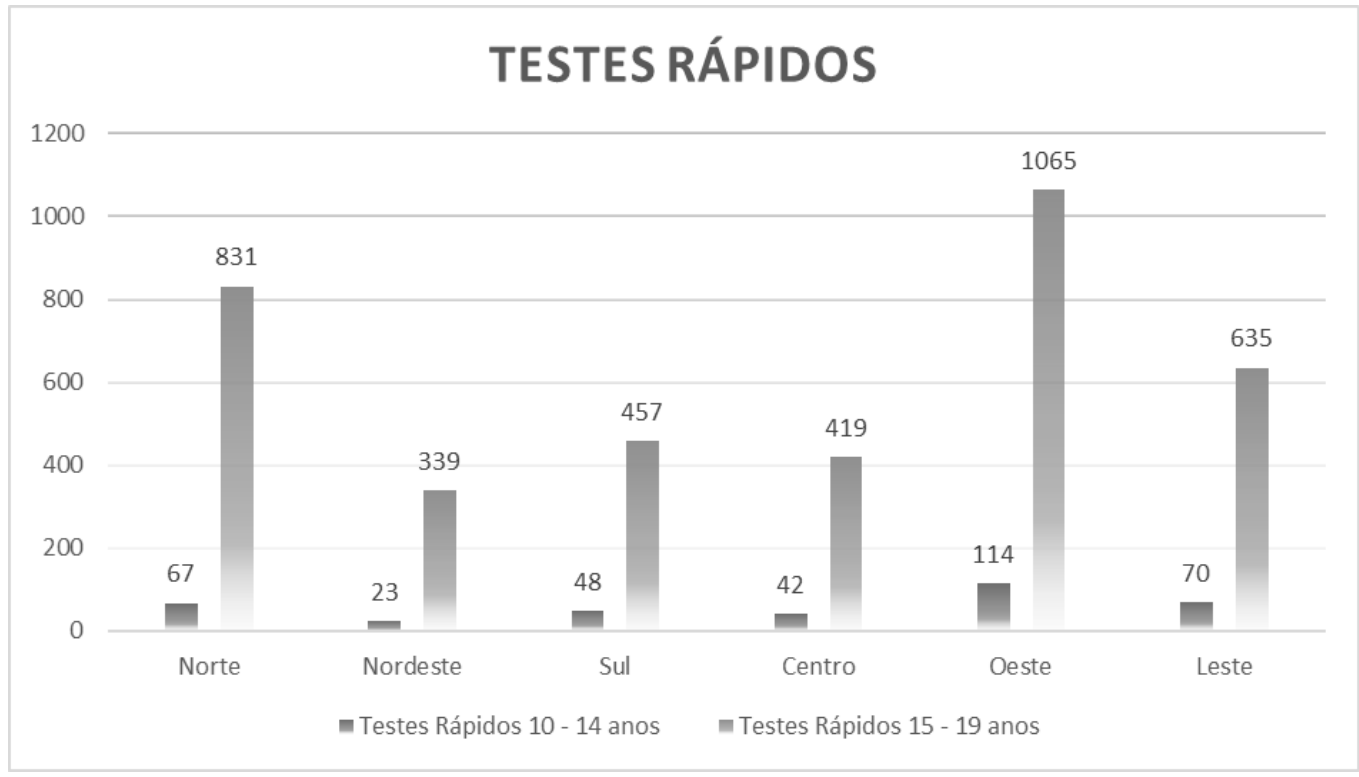

Fonte: Política de Saúde do Adolescente. 2020/2021. Santa Maria - RS.

Assim contabilizou 4.110 (100\%) testes rápidos realizados durante os 13 meses de análise, havendo predomínio do segundo grupo com 3.746 (91,14\%), de faixa etária dos 15 aos 19 anos. Em relação às regiões, os testes foram realizados em maior número nas regiões Oeste, com 1179 (28,69\%); Norte com 898 (21,85\%) e Leste com $705(17,15 \%)$ dos testes. Percebe-se que o segundo grupo de adolescentes ainda é maioria na procura de realização dos testes. Na região Oeste da cidade os locais que mais realizaram testagens nas duas faixas etárias foram a Unidade Básica de Saúde Ruben Noal responsável por 265 (22,48\%) testes e a Estratégia Saúde da Família Alto da Boa Vista por 231 (19\%), considerando o período da coleta de dados.

No estudo de Vieira (et al, 2021), se observou em uma amostra com 499 adolescente entre a faixa etária de 12 a 18 anos, que 471 (94,4\%) conheciam o preservativo masculino e $415(83,1 \%)$ o contraceptivo hormonal oral $(83,1 \%)$. Entretanto, ainda que minoria, $101(20,2 \%)$ indivíduos acreditavam que o contraceptivo oral protege contra infecções sexualmente transmissíveis e na 
pergunta seguinte se é necessário usar preservativo em toda a relação sexual $76(15,2)$ adolescentes acreditam que não preciso.

Os adolescentes têm algumas informações sobre saúde sexual, que acabam aprendendo através de amigos, internet entre outros, porém o assunto ainda é escasso ou mal-entendido por parte deles, como por exemplo o que significa sexualidade, e isto acaba acarretando riscos de adquirir IST's ou uma gravidez não planejada. Devendo ser um assunto mais abordado e enfatizado nas escolas e meios de saúde (MORAES, et al., 2019).

No que se dispõem de dados da vacinação contra HPV nos adolescentes, tem-se os seguintes números no gráfico 2:

Gráfico 2. Apresentação dos dados de imunização contra HPV com adolescentes, segundo a faixa etária e região geográfico-administrativa do município de Santa Maria - RS, 2020 - 2021.

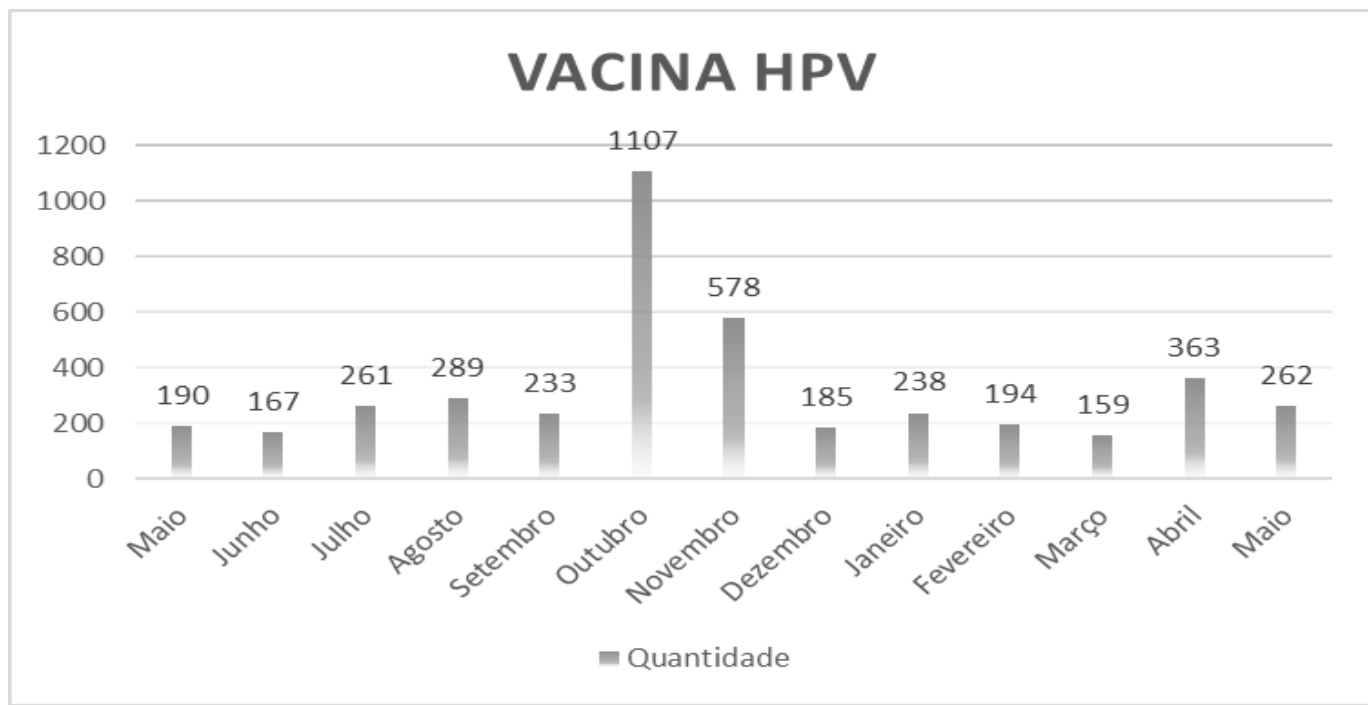

Fonte: Política de Saúde do Adolescente. 2020/2021. Santa Maria - RS.

Diante das $4.226(100,00 \%)$ aplicações de imunobiológicos durante os 13 meses de análise, o mês de outubro se destacou com 1.107 (26,19\%), depois novembro com 578 (13,68\%) de aplicações. Percebe-se que mesmo com a pandemia a imunização nos serviços contínuo ativa. O Papilomavírus Humano (HPV) dispõe mais de 150 tipos, sendo 40 deles oncogênicos, como principal fator de risco para neoplasias de colo uterino, e seu contágio ocorre por contado direto 
com os fluidos de indivíduo infectado, através de relações sexuais desprotegidas, como também ocorre pela transmissão vertical (mãe para filho durante o parto). A idade preconizada para se vacinar varia entre 9 e 14 anos para meninas e 11 a 14 anos para meninos, sendo administrada duas doses com intervalo de 6 meses entre elas (BRASIL, 2021).

Ainda se percebe uma escassez de informação por parte dos indivíduos maiores de 18 anos demostrada em uma amostra com 600 indivíduos, em que menos da metade $(40,1 \%)$ sabia dizer o que é o HPV e como se contrai (ABREU et al., 2018). Com isso, podendo ter porcentagens bem menores se formos entrevistar adolescentes abaixo de 18 anos. Com todas essas transições e descobertas, tornase necessária uma nova abordagem de produção de cuidado. Nesse contexto, a APS possui papel estratégico também na saúde do adolescente, uma vez que representa a principal porta de entrada da rede de atenção à saúde (RAS) para o desenvolvimento da assistência a população de forma integral (BRASIL, 2012)

Entretanto o cuidado com adolescentes é ainda um desafio para os profissionais, de que maneira abordar os assuntos e fazer eles se interessarem pela sua saúde e buscar informações e serviços de saúde. Tendo o ponto chave o acolhimento que deve ser realizado por todos os profissionais, desde a recepção e a entrada desse usuário no serviço (BRASIL,2017).

\section{CONCLUSÃO}

Portanto, percebe uma grande demanda de usuários entre a faixa etária dos 15 aos 19 anos na procura da realização de testes rápidos nos serviços de saúde, porém ainda se faz necessário um estudo mais aprofundado desses números para levantar a quantidade de testes com resultados positivos e negativos, o que poderá auxiliar os profissionais em ações contra IST's e reforçar a utilização de preservativos, pois sendo o único de protege contra infeções sexualmente transmissíveis.

$\mathrm{Na}$ questão de vacinação contra o Papilomavírus Humano, ainda é pouco disseminada a sua importância, devendo ter mais campanhas durante o ano, onde se nota que há mais públicos que procuram se imunizar e assim junto com o acolhimento ressaltar a importância dessa vacina. 


\section{AGRADECIMENTOS}

Primeiramente, quero agradecer ao projeto da bolsa PROBIC-UFN, intitulado como Educação, Saúde e Interprofissionalidade no Cenário Escolar: Integrando Ações de Ensino, Pesquisa e Extensão. A minha orientadora por todo aprendizado e crescimento que adquiri durante esse período. E a universidade franciscana por proporcionar essa experiência de agregação profissional.

\section{REFERÊNCIAS}

BRASIL. Ministério da Saúde. Caderno do gestor do PSE / Ministério da Saúde, Ministério da Educação. Brasília: Ministério da Saúde, 2015.

BRASIL. Ministério da Saúde. Conselho Nacional de Saúde. Resolução no 466, de 12 de dezembro de 2012. Aprova as diretrizes e normas regulamentadoras de pesquisas envolvendo seres humanos. Diário Oficial da União, Brasília, DF, 13 jun. 2013, p. 59.

BRASIL. Ministério da Saúde. Secretaria de Atenção à Saúde. Departamento de Ações Programáticas e Estratégicas. Proteger e cuidar da saúde de adolescentes na atenção básica [recurso eletrônico] / Ministério da Saúde, Secretaria de Atenção à Saúde, Departamento de Ações Programáticas e Estratégicas. Brasília: Ministério da Saúde, 2017.

BRASIL. Ministério da Saúde. Secretaria de Atenção à Saúde. Departamento de Atenção Básica. Política Nacional de Atenção Básica / Ministério da Saúde. Secretaria de Atenção à Saúde. Departamento de Atenção Básica. Brasília: Ministério da Saúde, 2012.

BRASIL. Portal do Governo Brasileiro. Campanha Vacinação HPV. Acesso em 09 jul 2021. Disponível em: https://portalarquivos.saude.gov.br/campanhas/vacinahpv/.

ABREU, M. N. S., et al. Conhecimento e percepção sobre o HPV na população com mais de 18 anos da cidade de Ipatinga, MG, Brasil. Ciência \& Saúde Coletiva, 23(3):849-860, 2018.

MORAES, A. L. et al. O adolescente e sua sexualidade: uma abordagem em educação e saúde na escola. Enferm Foco [Internet]10(2): 149-154, 2019.

POTTER, P. A. et al. Fundamentos da enfermagem. Ed. 9 - Rio de Janeiro: Elsevier, 2018. 
RIO GRANDE DO SUL. Secretária Estadual de Saúde, Departamento de Ações em Saúde, Seção de Saúde da Criança e do Adolescente. Política Estadual de Atenção Integral à Saúde de Adolescentes. 2010.

SADOCK, B. J; SADOCK, V. A. KAPLAN E SADOCK, Compêndio de psiquiatria, ciência do comportamento e psiquiatria clínica. Ed. 9. Porto Alegre: Artmed, 2007.

VIEIRA, K.J., et al. Conhecimentos de adolescentes sobre métodos contraceptivos e infecções sexualmente transmissíveis. Rev Baiana Enferm. 2021. Acesso em 28 set 2021. Disponível em: <http://www.revenf.bvs.br/pdf/rbaen/v35/1984-0446-rbaen-35e39015.pdf> 